Service social

\title{
La contribution des femmes gestionnaires du Cameroun à l'évolution des services sociaux
}

\section{Georgette Béliveau et Bernadette Ngo Nkouth}

Volume 44, numéro 1, 1995

Valeurs, pratiques, action sociale

URI : https://id.erudit.org/iderudit/706683ar

DOI : https://doi.org/10.7202/706683ar

Aller au sommaire du numéro

\section{Éditeur(s)}

École de service social de l'Université Laval

ISSN

1708-1734 (numérique)

Découvrir la revue

Citer cet article

Béliveau, G. \& Nkouth, B. N. (1995). La contribution des femmes gestionnaires du Cameroun à l'évolution des services sociaux. Service social, 44(1), 115-150. https://doi.org/10.7202/706683ar
Résumé de l'article

Cet article présente les résultats d'une recherche descriptive de type empirique selon l'approche qualitative, menée au Cameroun, sur la contribution des femmes gestionnaires à l'évolution des services sociaux dans ce pays. Après une brève référence aux principaux courants de recherche utilisés par les chercheurs pour étudier la question de la sous-représentation des femmes en gestion, les auteures présentent un nouveau type de recherche qui s'intéresse au style de gestion des femmes et à leur apport en administration. Les auteures exposent le déroulement de l'étude ainsi que la méthode de travail utilisée. Elles tracent ensuite le profil démographique des participantes, leur profil scolaire et leur cheminement de carrière. Les principaux résultats de l'étude portent sur la contribution des femmes gestionnaires à l'évolution de la culture organisationnelle, au style de gestion et au développement des services sociaux. Enfin, des liens pertinents sont établis avec le contexte des services sociaux au Québec. 


\section{CONTRIBUTION INTERNATIONALE}

\section{La contribution des femmes gestionnaires du Cameroun à l'évolution des services sociaux}

Georgette BÉLIVEAU

Professeure titulaire École de service social, Université Laval

Bernadette NGO NKOUTH

Étudiante au doctorat École de service social, Université Laval

Plusieurs études ont mis en relief le fait que les femmes de diverses professions sont sous-représentées dans l'exercice de fonctions de gestion. $C^{\prime} e s t$ d'ailleurs le cas en service social même si ce phénomène semble inconciliable avec le fait que les femmes sont majoritairement représentées. Au Québec par exemple, les femmes sont minoritaires dans les postes de la haute hiérarchie administrative au sein des établissements du réseau des services sociaux (Bradette, 1993: 11). Cette même tendance a été observée au Cameroun dans une récente analyse ${ }^{1}$ sur la situation des femmes dans les instances de prise de décision et plus spécifiquement en ce qui concerne le ministère des Affaires sociales et de la Condition féminine (MINASCOF) ${ }^{2}$ où les femmes gestionnaires représentent $39 \%$ de l'ensemble des responsables de ce département ministériel. 
De nombreuses études ont été réalisées pour expliquer cette sous-représentation des femmes en gestion. On s'est penché sur les obstacles qui entravent l'accès des femmes aux postes de gestion, sur les moyens à mettre en œuvre pour corriger cette situation et sur une meilleure connaissance des femmes gestionnaires et de leurs cheminements de carrière. De plus en plus, les analystes de la question commencent à s'intéresser au style de gestion des femmes et à leur apport en administration. Les travaux de Harel-Giasson et MarchisMouren (1988) soulignent la rareté des études traitant de ces questions. Toutefois, il se dégage des écrits sur le sujet que les contributions des femmes à la gestion des services sociaux pourraient porter sur trois volets : la culture organisationnelle, le style de gestion et le développement des services sociaux. II s'agit de contributions telles que décrites et écrites par les femmes gestionnaires. Notre recherche $s^{\prime}$ inscrit dans ce dernier courant. L'absence de données scientifiques sur les contributions des femmes à la culture organisationnelle et au développement des services sociaux suscite notre intérêt pour explorer ce phénomène auprès des femmes gestionnaires des services sociaux au Cameroun. La rareté de la littérature scientifique sur ce phénomène pose le problème de la référence à un modèle théorique sur lequel notre étude pouvait s'appuyer. Il a été jugé opportun, pour I'observation de ce phénomène, de mener une recherche empirique de nature exploratoire, selon l'approche qualitative.

Cette recherche est une étude de cas dont le but visé est l'exploration et l'approfondissement des connaissances du phénomène de l'apport des gestionnaires du réseau des affaires sociales. Elle vise principalement à décrire les contributions des femmes gestionnaires à l'évolution de la culture organisationnelle, à l'amélioration du style de gestion et au développement des services sociaux au Cameroun.

\section{Les écrits sur le sujet}

Les écrits consultés portent sur les femmes et la gestion en général, ainsi que sur les femmes et la gestion des services sociaux en particulier. Ces études traitent principalement de la discrimination sexuelle envers les femmes gestionnaires, des barrières limitant leur accès et leur mobilité professionnelle en gestion, de leur compétence, des facteurs de réussite, des moyens et des stratégies utilisés par les femmes gestionnaires pour se maintenir en fonction. Quelques études portent sur un nouveau courant de recherche qui s'intéresse à l'apport particulier éventuel des femmes gestionnaires dans les organisations. À des fins de synthèse, on a regroupé ces écrits dans trois thèmes : 
la situation personnelle et sociale des femmes, les facteurs organisationnels et le nouveau courant de recherche. Ce dernier thème, privilégié dans le présent article, soutient que ce sont à la fois le sexe, l'environnement organisationnel ainsi que le système social et institutionnel dans lequel s'intègrent les organisations qui définissent et influencent le comportement des femmes au travail (Fagenson, 1990).

\section{Le nouveau courant de recherche}

D'après Harel-Giasson (1990), "les femmes cadres représentent un groupe qui a des caractéristiques propres le distinguant du groupe qui a jusqu'à maintenant, de façon quasi exclusive, donné le ton aux organisations. Cela constitue un capital potentiel pour introduire de nouvelles façons de penser, de parler et de faire». Selon la même auteure, les héritages de la culture féminine supposent effectivement un apport distinctif des femmes gestionnaires dans les organisations. Elle précise : «...Cet apport se conjugue avec une zone de conformité à la culture des cadres masculins. Pour cette raison, la culture féminine influencera vraisemblablement les entreprises plutôt sous forme de "traces" que de changements radicaux, du moins dans un avenir prévisible» (Harel-Giasson, 1990). II ressort que ces « traces » varieront en fonction des caractéristiques des organisations, du nombre et de la proportion de femmes dans les organisations, du niveau hiérarchique et du type de responsabilités (centrales ou périphériques), des habiletés personnelles, de l'âge d'une femme gestionnaire et de la longueur de son expérience dans une fonction et de l'appartenance à des réseaux de femmes gestionnaires (Harel-Giasson, 1990).

Par ailleurs, une revue des écrits sur l'évolution du service social a permis d'identifier les gestes posés par les femmes gestionnaires des services sociaux pour améliorer ou développer ces derniers. Ces gestes apparaissent sous forme d'expériences de travail décrites ou écrites par les femmes gestionnaires. Il a été possible de relier ces contributions à l'un ou l'autre des trois volets que comporte notre étude, à savoir la culture organisationnelle, le style de gestion ou le développement des services sociaux. Les sections suivantes présentent une synthèse des apports des femmes gestionnaires à partir de ces trois volets.

\section{La culture organisationnelle dans les services sociaux}

On a regroupé sous le volet culture organisationnelle tout ce qui concerne la personnalité d'une organisation et détermine ses orientations 
(Bergeron, 1989: 133). Dans le cas des services sociaux, il s'agit principalement des valeurs privilégiées et qui déterminent en grande partie les politiques, les plans et le fonctionnement du système des services sociaux (Guberman et al.,1994; Poulin, 1982). La synthèse des écrits sur le volet culture organisationnelle s'organise autour du concept "valeurs».

D'une manière générale, les écrits sur l'histoire des services sociaux en France, au Canada et plus spécifiquement au Québec révèlent que la culture organisationnelle des services sociaux a évolué au cours des époques en fonction des valeurs privilégiées par la société. Dans ces trois contextes, les valeurs qui émergent des écrits consultés sont les suivantes: la charité chrétienne, la participation, I'assistance, l'aide sociale, la sécurité sociale, la justice sociale, l'égalité des chances et l'accessibilité aux services, la solidarité humaine ou la philanthropie, I'action sociale (Hardy, 1994 ; Guest, 1993; rapport Rochon, 1988; Perron, 1986; Poulin, 1982; Guerrand et al., 1978).

Pour ce qui est de l'évolution des services sociaux au Cameroun, il ressort de l'analyse de Emgbang (1970) que les mutations sociales survenues dans ce pays sont à l'origine de la création des institutions capables de résorber les conséquences sociales inhérentes à ces changements. On assiste à une rupture entre la communauté traditionnelle et l'individu-cette communauté qui, jadis, se chargeait de la résolution des problèmes sociaux vécus par ses membres. En 1950 l'administration coloniale a créé le Service des Affaires sociales et de la Population qui succédait au service du Bien-être, susceptible d'aider les populations à s'adapter aux institutions nouvelles tout en conservant un lien avec leur passé culturel. Sa mission fondamentale était alors l'éducation et l'assistance matérielle ou morale aux populations défavorisées. La récession économique a imposé à l'État de réorienter sa politique en matière d'organisation des services sociaux. C'est alors qu'est apparue la notion d'assistance-participation.

À la lumière de ce qui précède, on remarque que dans les quatre contextes considérés il y a eu évolution de la culture organisationnelle des services sociaux marquée par différentes valeurs. Dans tous les cas, la charité chrétienne apparaît comme le point de départ de I'organisation des services sociaux. Cette évolution s'est traduite par l'existence des moyens traditionnels (les familles, la communauté ou les mouvements religieux) de résolution des problèmes sociaux, suivie d'une intervention de l'État qui assure une prise en charge de l'organisation des services sociaux (en privilégiant certaines valeurs) 
et on assiste de nos jours au développement d'un partenariat entre l'État et le secteur privé dans ce domaine. Dans la présente recherche, nous avons retenu les cinq principales valeurs ayant sous-tendu I'organisation des services sociaux au cours des époques dans les quatre contextes: la charité chrétienne, la participation, la justice sociale, l'égalité des chances et l'accessibilité, la philanthropie. Quel sens donner à ces valeurs?

Au Canada et au Québec, I'organisation des services sociaux, basée sur la charité chrétienne au début des années 50, signifie que ce sont les individus, les familles, les voisins et l'Église qui prennent en charge la résolution des problèmes sociaux (Guest, 1993; Perron, 1986 ; Poulin, 1982). En France, I'organisation des services sociaux dès leur apparition est fortement marquée par la valeur charité chrétienne qui se manifeste à travers une multitude d'œuvres de bienfaisance (Pascal et De Robertis, 1994; Guerrand et al., 1978), alors qu'au Cameroun c'est la famille élargie (la communauté) qui prend en charge la résolution des problèmes sociaux.

La valeur "participation» correspond à l'engagement des usagers dans I'organisation des services sociaux au Canada comme au Québec. Cet engagement se traduit par la création de structures organiques de participation, par la mise sur pied de comités d'usagers et par la participation des usagers aux conseils d'administration des établissements (Guest, 1993 ; Poulin, 1982; rapport CastonguayNepveu, 1972). En France, la valeur "participation" renvoie à une nouvelle impulsion donnée à la dimension de la relation avec l'usager pour en arriver à la notion de partenariat. Cette impulsion s'est traduite par la mise en place des mesures prévoyant l'exercice du droit des usagers à l'égard de l'administration, en leur donnant la possibilité d'une plus grande participation aux décisions qui les concernent (Périnel, 1994). Au Cameroun, les documents du Ministère ${ }^{3}$ mentionnent plutôt l'assistance-participation. Celle-ci se traduit par une aide morale, matérielle ou financière aux personnes ayant des problèmes sociaux et, en même temps, par une implication et une participation des bénéficiaires à la résolution de leurs problèmes.

Au Canada et plus spécifiquement au Québec, la justice sociale signifie que l'organisation des services sociaux incombe à la société entière, donc à l'État (Guest, 1993; rapport Rochon, 1988). En France, bien que les écrits soient peu explicites sur le sujet, l'idée de justice sociale se traduit par la généralisation de la Sécurité sociale à l'ensemble de la population (Périnel, 1994).

En ce qui regarde le principe d'égalité des chances et d'accessibilité, il renvoie au caractère universel et gratuit des services 
sociaux au Canada et au Québec (Guest, 1993; rapport Rochon, 1988). Dans le contexte français, I'analyse de Périnel (1994) révèle que l'évolution de la protection sociale s'est traduite, notamment, par une meilleure répartition des équipements et des services sur le territoire, de même que par une amélioration de leur qualité. L'accessibilité prend la même forme au Cameroun et se traduit par la décentralisation des services sociaux dans les régions pour rapprocher les services des usagers (décret $n^{\circ} 88 / 1281,1988$ ).

En ce qui concerne la philanthropie, elle se développe au Québec à partir du discours de l'État qui prône le partage des responsabilités entre lui et la communauté, ce qui entraîne une résurgence des organismes communautaires (rapport Rochon, 1988; Mayer et Groulx, 1985). La philanthropie prend à peu près la même signification au Cameroun. Elle correspond à l'incitation du secteur privé à s'impliquer dans l'organisation des services sociaux. Dans le contexte français, la littérature souligne l'importance du secteur privé dans le domaine des services sociaux (Hardy, 1994 ; Picard, 1994).

L'étude des valeurs est donc la dimension retenue pour étudier la variable culture organisationnelle. Les principales valeurs ayant guidé l'organisation et la distribution des services sociaux au Québec sont: la charité chrétienne, la participation, la justice sociale, l'égalité des chances et d'accessibilité aux services, la solidarité humaine ou la philanthropie. Au Cameroun, la culture organisationnelle du réseau des affaires sociales a été tour à tour sous-tendue par la charité chrétienne, l'assistance, l'assistance-participation, la justice sociale, l'accessibilité et la philanthropie. L'ordre d'apparition des valeurs suit la logique d'évolution qui émerge de la littérature en partant des plus anciennes à celles qui sont d'actualité. Ces six valeurs sont les indicateurs utilisés pour l'étude qui veut vérifier si elles ont réellement sous-tendu la culture organisationnelle des services sociaux au Cameroun et décrire les contributions des femmes gestionnaires à l'évolution de cette culture. Le tableau 1 présente les indicateurs utilisés pour "opérationnaliser» la dimension "valeurs».

TAbleau 1

Variable : la culture organisationnelle

Dimensions

Les valeurs
Indicateurs

Charité chrétienne

Assistance

Assistance-participation

Justice sociale

Accessibilité

Philanthropie 


\section{Le style de gestion}

Dans le cadre de notre étude, le style de gestion fait référence au processus classique de gestion qui comprend quatre fonctions: Ia planification, l'organisation, la direction et le contrôle (Bergeron, 1989 ; Crener et Monteil, 1980; Koontz et O'Donnell, 1980). En tenant pour acquis que les théories en matière de gestion sont quasi universelles, on estime que ce modèle répond aux besoins de l'étude. D'après l'étude de Bradette (1993), les femmes ont à innover et à créer leur propre modèle de gestion.

La planification concerne la sélection des objectifs, la prévision des moyens pour les atteindre et l'élaboration des plans (Béliveau, 1992). Au Québec, les femmes gestionnaires des services sociaux ont élaboré des modèles de gestion et des plans d'organisation des services (Leczinska, 1982; Berlinguet, 1968) ou rédigé des documents critiques et fait des propositions pour améliorer les plans d'organisation (Boissinot, 1976). En France, les femmes gestionnaires des services sociaux ont inventé la réforme du service social des directions de l'action sociale et sanitaire (Braquehais, 1994).

La fonction «organisation» consiste à réaliser des plans en fonction des objectifs à atteindre, des capacités et des centres d'intérêt des individus. Cette fonction nécessite la définition des tâches et la précision des relations d'autorité (Béliveau, 1992). Les femmes gestionnaires des services sociaux au Québec ont ainsi procédé à la définition des tâches au sein du réseau des services sociaux (Berlinguet, 1968). Elles ont proposé une conciliation des fonctions du service social et celles de I'assistance publique (Boissinot, 1956: 24-26). Elles ont fait des suggestions portant sur l'attitude à adopter par les travailleurs sociaux dans le nouveau cadre de distribution des services sociaux issu de la réforme (Blouin, 1994). En France, les femmes gestionnaires des services sociaux ont pris une part très active dans la réorganisation des services sociaux publics régionaux (Braquehais, 1994).

La fonction de direction relève principalement des relations interpersonnelles: elle nécessite du gestionnaire qu'il puisse communiquer avec les membres de son unité organisationnelle et améliorer les conditions de travail en donnant aux personnes plus de responsabilités et d'autorité (Bergeron, 1989). L'étude de Chernesky et Bombyk (1988) arrive à la conclusion que les femmes gestionnaires ont un style de leadership plus humain et différent de celui des hommes. Elles sont perçues comme ayant des compétences en communication interpersonnelle: écoute, empathie, ouverture, souplesse et participation (Guberman et al., 1994 ; Chernesky et Bombyk, 1988). 
Au Québec, les femmes gestionnaires ont conçu et développé un style de leadership adapté aux services sociaux qu'elles dirigent (Leczinska, 1982).

Contrôler, c'est mesurer les résultats actuels, comparer les résultats aux standards, prendre des mesures correctives (Béliveau, 1993). Les contributions des femmes gestionnaires à cette fonction sont peu perceptibles dans les écrits consultés. Néanmoins, il ressort des résultats du prétest réalisé par une des auteures que certaines femmes gestionnaires des services sociaux au Québec ont conçu et développé des outils d'évaluation des services et des programmes (Ngo Nkouth, 1994).

Dans le cadre de cette étude, seules les fonctions de direction et de contrôle sont retenues pour l'étude du modèle de gestion et de la nature des contributions des femmes à l'amélioration du style de gestion. On estime que la fonction planification sera abordée par les femmes sous l'angle de "culture organisationnelle». Quant à la fonction organisation, elle est abordée dans la section sur le développement des services sociaux où il sera question des types d'organisations et de la nature des services.

"La direction est une activité de leadership à travers laquelle le gestionnaire se sert de tous les aspects de la motivation et de la communication pour amener ses collaborateurs à effectuer leurs tâches quotidiennes avec intérêt, tout en tenant compte des plans et des objectifs communs"(Bergeron, 1989: 5 et 344). Le même auteur distingue cinq styles de leadership: les styles autocratique, paternaliste, démocratique, collégial et le laisser-faire. "Le gestionnaire autocratique commande, prend toutes les décisions et demande à ses subordonnés de faire leur travail exactement comme il le désire " (Bergeron, 1989: 347). "Le gestionnaire paternaliste a une attitude bienveillante et charitable envers ses subordonnés. II désire leur offrir les meilleurs salaires, de bonnes conditions de travail, et d'autres avantages [...]. Il est généreux et multiplie les moyens d'améliorer le sentiment de sécurité chez les employés " (Bergeron, 1989: 348). "Le gestionnaire démocratique encourage la participation des subordonnés à la prise de décision [...]. Ce style de leadership est caractérisé par la délégation de l'autorité, par la participation des employés au processus décisionnel et par la liberté d'effectuer les tâches à leur manière »(Bergeron, 1989: 348). "Le style collégial ressemble à l'approche précédente [...]. Une place prépondérante est accordée à la loyauté, à l'amitié et à l'échange [...]. Ils aiment plutôt travailler en équipe et sont conscients du niveau de qualification, de compétence et d'intérêt de ceux avec qui ils travaillent. Chacun est considéré comme un collègue»(Bergeron, 1989: 349). "Le gestionnaire qui 
opte pour le laisser-faire n'a que peu d'intérêt pour sa tâche et ses subordonnés. Il laisse une liberté totale à ces derniers en ce qui a trait au choix des objectifs et à leur réalisation" (Bergeron, 1989: 349).

Le but principal de la dimension "direction» est de rendre compte des formes de motivation du personnel, du style de communication, de participation du personnel et du style de leadership que les femmes gestionnaires favorisent dans les organisations qu'elles dirigent pour améliorer la gestion des services sociaux au Cameroun. Le tableau 2 regroupe les indicateurs utilisés pour explorer l'apport des femmes à l'amélioration de la gestion.

Le contrôle est une procédure qui permet au gestionnaire d'évaluer sa performance en comparant les résultats obtenus aux objectifs et de prendre les mesures appropriées pour remédier aux situations défavorables (Bergeron, 1989). Le gestionnaire doit s'assurer que les ressources sont utilisées à bon escient. La fonction "contrôle " lui permet donc de faire une évaluation en utilisant différents outils, tels que la budgétisation et la vérification. Le gestionnaire doit évaluer périodiquement la performance des personnes, vérifier si leurs capacités sont exploitées au maximum et relever leurs forces et leurs faiblesses (Bergeron, 1989). Parmi les techniques de contrôle, on note le contrôle de la gestion des ressources humaines, des ressources financières, des ressources physiques et matérielles, des projets (Bergeron, 1989). Le but de la dimension de contrôle est de rapporter la description faite par les femmes gestionnaires des mécanismes d'évaluation de la performance des personnes, de contrôle des ressources financières, d'évaluation des programmes et des innovations en matière de services sociaux. Le tableau 2 présente les indicateurs utilisés pour "opérationnaliser» cette dimension.

\section{Tableau 2}

\section{Variable : style de gestion}

\begin{tabular}{ll}
\hline Dimensions & Indicateurs \\
\hline Direction & a) Formes de motivation du personnel \\
& b) Style de communication privilégié avec le personnel \\
& c) Formes de participation \\
d) Style de leadership privilégié : & - autocratique \\
& - paternaliste \\
& - démocratique \\
& - collégial \\
& - laisser-faire \\
Contrôle & a) Évaluation de la performance des personnes \\
& b) Contrôle des ressources financières \\
c) Évaluation des programmes & d) Évaluation des innovations dans le domaine des services sociaux
\end{tabular}




\section{Le développement des services sociaux}

Que ce soit au Canada, au Québec ou en France, le développement des services sociaux vise le développement des organismes de distribution, des services offerts, des ressources financières et des agents d'intervention (Guest, 1993; Rochon, 1988; Poulin, 1982; Guerrand et al., 1978).

Selon les époques, trois types d'organismes ont distribué des services sociaux au Québec: des organisations privées d'avant la réforme des services sociaux de 1971, des organisations publiques d'après la réforme et des organismes communautaires (Guest, 1993; Rochon, 1988; Poulin, 1982). La même tendance est observée en France où l'on souligne que les services sociaux ont été organisés à leurs débuts par les œuvres privées de bienfaisance (Picard, 1994) et appuyées par les organisations publiques (Hardy, 1994). Par la suite, certaines œuvres privées se sont transformées en organisations publiques (Guerrand et al., 1978). Dans le contexte camerounais, deux types d'organismes de distribution émergent des écrits: les œuvres sociales privées, puis les organisations publiques (Emgbang, 1970).

L'apport des femmes gestionnaires au développement des organismes de distribution des services sociaux est peu traité dans les écrits consultés. Néanmoins, au Québec comme en France, les femmes ont créé, dirigé et développé plusieurs organisations privées ou œuvres de bienfaisance. Elles ont pris part aux comités d'implantation ou de transformation des agences privées en établissements publics de services sociaux (Braquehais, 1994 ; Picard, 1994 ; Paré, 1988; Guerrand et al., 1978).

La nature des services offerts a évolué en fonction des époques et du secteur d'intervention. Au Québec, Rochon (1988) et Poulin (1982) identifient plusieurs catégories de services: services généraux ou traditionnels, services spécialisés, services ciblés sur les clientèles et services répondant à des problématiques particulières, telles que la violence faite à la personne, la mésadaptation sociale et l'intégration sociale. En France, on peut citer les catégories suivantes: services généraux axés sur la famille, services d'éducation et d'assistance sociales et ceux visant le développement de formes de mutualités (Picard, 1994; Braquehais, 1994; Guerrand et al., 1978). Au Cameroun, plusieurs types de services sont organisés: services généraux, services spécialisés, services ciblés sur des clientèles et services répondant à des problématiques particulières ou ponctuelles. Les contributions des femmes gestionnaires au développement des services offerts en France ou au Québec se résument à l'organisation, à la mise en place ou à l'amélioration d'une ou de plusieurs de ces 
catégories (Braquehais, 1994 ; Picard, 1994 ; Paré, 1988 ; Guerrand et al., 1978).

Au Québec, on relève deux sources de financement des services sociaux: financement public provenant de l'État et financement privé, assuré par les campagnes de financement (Guest, 1993 ; Poulin, 1982). En France, les services sociaux sont financés principalement par les régimes de Sécurité sociale, l'État et les collectivités territoriales. On retrouve donc deux sources de financement, comme dans le contexte québécois. Les femmes gestionnaires ont participé activement à I'organisation des campagnes de financement des œuvres (Paré, 1988; Guerrand et al., 1978) et elles ont contribué au maintien des budgets de l'État par l'élaboration des budgets pertinents et par une gestion saine (Ngo Nkouth, 1994). Au Cameroun, en plus des sources de financement identifiées ci-dessus, on révèle un troisième mode de financement des services sociaux, à savoir le financement international.

L'analyse des écrits permet d'identifier trois catégories d'agents d'intervention: les bénévoles, les professionnels et les technocrates (Pascal et De Robertis, 1994 ; Mallet, 1994 ; Poulin, 1982).

Les gestionnaires québécoises ont contribué au développement des ressources humaines dans ce domaine. Certaines d'entre elles ont donné une impulsion importante à la formation des travailleurs sociaux en prenant une part active à la formation, en faisant la supervision de stagiaires, en collaborant à l'élaboration et à la révision des programmes de formation collégiale et universitaire (Ngo Nkouth, 1994 ; Paré, 1988). En France, les femmes gestionnaires des services sociaux ont été des pionnières en matière de formation des intervenants sociaux. Elles ont mis sur pied des écoles de formation et ont aussi pris part à la réorganisation et à l'adaptation des programmes de formation dans ces écoles (Guerrand et al., 1978; Pascal et al., 1994).

Les quatre aspects du développement des services sociaux pertinents pour l'étude et qui constituent les dimensions étudiées sont: les types d'organisations, la nature des services offerts, les ressources financières et les ressources humaines.

Une organisation de services sociaux est un établissement public ou privé qui fournit des services sociaux. Au Cameroun, le MINASCOF englobe l'ensemble des organisations publiques. II a une administration centrale et des services extérieurs. L'administration centrale comprend un secrétariat général et cinq directions : la direction des affaires générales, la division des études, de la planification et de l'évaluation, la direction de la promotion féminine, la direction de la réinsertion sociale, la direction de la protection de l'individu et 
de la famille. Ces directions sont constituées de sous-directions et de services. Les services extérieurs comprennent les services à compétence territoriale, les unités techniques de base, les établissements et organismes spécialisés ${ }^{4}$. Les organisations privées sont essentiellement des œuvres sociales privées, créées et dirigées par des particuliers. Placées sous la tutelle du MINASCOF, leurs activités doivent s'inscrire dans le champ des services sociaux.

L'étude veut rendre compte des expériences de travail, telles que rapportées par les femmes gestionnaires en matière de développement des organismes de distribution. Dans une perspective d'évolution des organismes de distribution, on décrit les contributions des femmes gestionnaires au développement des types d'organisations de services sociaux. Le tableau 3 présentera les indicateurs utilisés pour "opérationnaliser» cette dimension.

La nature des services renvoie aux types de services offerts à la clientèle. Au Cameroun, les services généraux ou traditionnels sont des services sociaux de base fournis dans les organisations publiques et dans certaines œuvres sociales privées: l'aide psychosociale, l'assistance financière aux indigents et aux personnes nécessiteuses, I'assistance matérielle et financière aux filles mères en difficulté, I'assistance médicale, l'aide à la famille, la sauvegarde de la petite enfance, etc. Les services spécialisés sont des services organisés pour une clientèle qui demande un suivi particulier: éducation spéciale et rééducation fonctionnelle en faveur des personnes handicapées, éducation en milieu ouvert et rééducation en internat pour les jeunes délinquants, etc. Les services ciblés sur la clientèle sont des services organisés pour une clientèle identifiée de façon précise: services d'éducation féminine, services d'intégration économique de la femme, services de réinsertion socio-économique des personnes handicapées, services d'assistance à la famille et de protection de l'enfance, etc. Les services répondant à une problématique spécifique concernent des problèmes ponctuels ou particuliers pour lesquels on organise des services afin de les juguler: lutte contre les fléaux sociaux (prostitution, alcoolisme, toxicomanie), action démographique (parenté responsable, intégration des populations marginales).

La dimension "nature des services » rapporte les expériences de travail, décrites par les femmes en ce qui concerne l'organisation et le développement des services offerts dans les quatre catégories de services décrites ci-dessus. Le tableau 3 présentera les indicateurs utilisés pour "opérationnaliser » cette dimension.

Les ressources financières renvoient aux différentes sources de financement des services sociaux. Au Cameroun, on relève trois 
modes de financement des services sociaux: le financement gouvernemental, privé et international. La première source est constituée des budgets que l'État alloue annuellement au MINASCOF pour réaliser sa mission. Le financement privé concerne les fonds engagés par des particuliers dans la création et le fonctionnement des œuvres sociales privées. Les fonds privés proviennent aussi des organisations non gouvernementales nationales. Pour ce qui est des sources de financement international, elles représentent les fonds accordés par les organisations non gouvernementales internationales pour financer certains projets sociaux du MINASCOF.

Les trois sources de financement sont importantes, et l'étude les retient. II s'agit de rendre compte de la description faite par les femmes gestionnaires de leurs expériences de travail pour maintenir ou accroître les budgets de leur organisation. Le tableau 3 mentionnera les indicateurs permettant l'opérationalisation de cette dimension.

Le développement des ressources humaines renvoie au développement des capacités des agents d'intervention indispensables à la distribution des services sociaux. II englobe tous les aspects liés à la formation. Plusieurs types d'agents d'intervention, avec ou sans formation spécifique, sont intervenus dans la distribution des services sociaux au Québec. Il s'agit: des bénévoles sans formation particulière; des aides sociaux ou assistants sociaux diplômés des établissements de formation en assistance sociale; des techniciens en assistance sociale formés dans des collèges d'enseignement général et professionnel; des travailleurs sociaux professionnels formés dans des écoles de service social universitaires; des autres groupes professionnels ayant une formation universitaire.

Au Cameroun, le réseau des affaires sociales emploie des professionnels en travail social et des personnes formées dans d'autres disciplines. Ces dernières suivent un stage d'imprégnation au moment de leur recrutement. Toutefois, on estime que l'effectif du Ministère chargé des affaires sociales se compose en grande partie de travailleurs sociaux professionnels dont le corps est constitué par:

- les inspecteurs et inspecteurs principaux des affaires sociales, qui sont des professionnels ayant une formation universitaire ou équivalente ;

- les assistants et assistants adjoints des affaires sociales, qui sont des intervenants de niveau intermédiaire formés à l'École nationale des Assistants des Affaires sociales (ENAAS). 
L'étude veut rendre compte des expériences de travail décrites par les femmes gestionnaires pour développer les capacités des personnes. Deux types d'agents d'intervention sont utilisés comme des indicateurs pour l'étude de la dimension "ressources humaines": inspecteurs des affaires sociales et assistants des affaires sociales. Le tableau 3 qui suit regroupe les indicateurs utilisés pour opérationnaliser la dimension "ressources humaines ». Il comprend aussi les trois dimensions définies précédemment, à savoir les types d'organisations, les services offerts et les ressources financières.

Tableau 3

Variable : développement des services sociaux

\begin{tabular}{|c|c|}
\hline Dimensions & Indicateurs \\
\hline Type d'organisations & $\begin{array}{l}\text { a) publiques } \\
\text { b) privées }\end{array}$ \\
\hline Nature des services & $\begin{array}{l}\text { a) services généraux ou traditionnels } \\
\text { b) services spécialisés } \\
\text { c) services ciblés sur la clientèle } \\
\text { d) services répondant à une problématique particulière }\end{array}$ \\
\hline $\begin{array}{l}\text { Les ressources } \\
\text { financières }\end{array}$ & $\begin{array}{l}\text { Les sources de financement: } \\
\text { a) gouvernementale } \\
\text { b) privée } \\
\text { c) internationale }\end{array}$ \\
\hline $\begin{array}{l}\text { Les ressources } \\
\text { humaines }\end{array}$ & $\begin{array}{l}\text { a) inspecteurs et inspecteurs principaux des affaires sociales } \\
\text { b) assistants et assistants adjoints des affaires sociales }\end{array}$ \\
\hline
\end{tabular}

Les éléments pertinents répertoriés ci-dessus serviront à cerner les contributions des femmes gestionnaires des services sociaux. Les variables d'analyse, de culture organisationnelle, de style de gestion et le développement des services sociaux nous ont permis d'identifier les dimensions de l'objet de l'étude. Elles nous ont aussi amenées à adopter un cadre $d^{\prime}$ analyse qui s'inspire $d^{\prime}$ un modèle classique de gestion et s'appuie sur le modèle québécois de l'évolution des services sociaux, pour finalement arriver à élaborer un cadre $d^{\prime}$ analyse adapté au contexte camerounais. C'est à partir des dimensions et des indicateurs que l'instrument de collecte des données a été élaboré.

On ne saurait clore ce chapitre sans préciser la notion de femmes gestionnaires. La plupart des ouvrages portant sur la gestion utilisent les termes manager, gestionnaire, directeur, dirigeant, cadre. En recoupant le sens que ces ouvrages donnent à ces différents termes, il apparaît que ces termes désignent une même personne qui, au sein d'une organisation, doit coordonner et intégrer les personnes, 
les activités et les fonctions, en vue d'atteindre les buts fixés par I'organisation (Bergeron, 1989; Koontz et al., 1980; Crener et al., 1980). Les gestionnaires se retrouvent à différents paliers au sein $d^{\prime}$ 'une organisation. Vue sous l'angle vertical, on distingue les cadres inférieurs qui coordonnent les activités des employés, les cadres intermédiaires qui dirigent les services, les cadres supérieurs qui coordonnent toutes les activités de l'organisation (Bergeron, 1989).

La fonction de gestionnaire est souvent associée à la notion de pouvoir qui se traduit par deux formes d'autorité: l'autorité hiérarchique et l'autorité fonctionnelle. Les personnes chargées d'accomplir des tâches directement reliées à la mission de I'organisme exercent une autorité hiérarchique et les personnes qui élaborent les politiques et les procédures exercent une autorité fonctionnelle (Bergeron, 1989).

L'étude considère comme gestionnaires de niveau inférieur les femmes "gestionnaires de premier niveau ", qui ont sous leur responsabilité des intervenants sociaux fournissant des services à la clientèle. II s'agit de femmes exerçant une fonction de chef de service. Les gestionnaires de niveau intermédiaire sont des directrices de services. Elles ont sous leur responsabilité des chefs de services, c'est-à-dire des gestionnaires de premier niveau. Dans la présente étude, les femmes gestionnaires de niveau intermédiaire occupent un poste de directrice ou assimilé dans les services centraux ou extérieurs du MINASCOF. Quant aux femmes gestionnaires de niveau supérieur, elles ont la responsabilité de coordonner toutes les activités d'un organisme autonome. Elles exercent une fonction de directrice d'un établissement spécialisé du MINASCOF ou d'une œuvre sociale privée. Les femmes gestionnaires fonctionnelles sont responsables de I'élaboration des politiques et du contrôle au MINASCOF. Il s'agit des femmes occupant un poste d'inspecteur général, de conseiller technique ou de chef de la division des études au MINASCOF.

En résumé, la notion de femmes gestionnaires renvoie aux fonctions suivantes: gestionnaire de premier niveau, de niveau intermédiaire, de niveau supérieur ou fonctionnelle.

\section{Le déroulement de l'étude et la méthode de travail}

Le but de l'étude que nous décrivons est d'approfondir les connaissances sur l'apport des femmes gestionnaires à l'évolution de la culture organisationnelle, à l'amélioration du style de gestion et au développement des services sociaux au Cameroun. II s'agit d'une recherche descriptive de type empirique selon I'approche qualitative, dont le but visé est l'exploration et l'approfondissement des connais- 
sances du phénomène de l'apport des femmes gestionnaires des services sociaux. La méthode de travail s'inspire du modèle de Yin (1989): il s'agit d'un devis d'étude de cas multiples (10 cas) avec plusieurs unités d'analyse. On s'est aussi inspiré des travaux de Dorais (1993) et de Gauthier (1984) pour élaborer cette méthode de travail.

L'étude a été menée auprès de dix femmes gestionnaires du réseau des affaires sociales au Cameroun. La sélection des répondantes s'est faite sur deux critères: travailler au ministère des Affaires sociales et de la Condition féminine ou dans une œuvre sociale privée et occuper un poste de gestionnaire fonctionnelle ou des niveaux supérieur, intermédiaire et inférieur. La formation en service social n'était pas considérée parmi ces critères, car le personnel exerçant dans le réseau des affaires sociales au Cameroun est constitué de professionnels formés en service social ou dans une autre discipline. Une méthode d'échantillonnage non probabiliste a été privilégiée, étant donné que l'étude ne vise pas la généralisation des résultats à l'ensemble des femmes gestionnaires du réseau des affaires sociales au Cameroun. La méthode de l'échantillon typique a été utilisée. "Il s'agit en fait de privilégier, autant que I'on puisse le faire, des unités typiques ou encore des personnes qui répondent au «type idéal » par rapport aux objectifs de la recherche » (Mayer et Ouellet, 1991 : 389). L'échantillon a été formé à partir de contacts directs et personnels. La répartition catégorielle selon la position occupée et le milieu d'intervention est la suivante:

\section{TABleau 4}

\section{Les cadres ayant participé à l'étude}

\begin{tabular}{l} 
Nombre et type de cadres \\
\hline-2 gestionnaires de premier niveau \\
-3 gestionnaires de niveau intermédiaire \\
-1 gestionnaire de niveau supérieur \\
-2 gestionnaires de niveau supérieur \\
-2 gestionnaires fonctionnelles
\end{tabular}

Genre d'organisation

- Ministère

- Ministère ou services extérieurs

- Institution spécialisée du ministère

- OEuvres sociales privées

- Ministère

La collecte des données s'est effectuée pendant les mois de novembre et de décembre 1994 à Yaoundé au Cameroun. Un prétest avait été effectué à l'hiver 1994 auprès de quatre femmes gestionnaires des services sociaux à Québec. Cette étape s'est avérée importante, puisqu'on se trouvait en présence d'un nouveau courant de recherche et en l'absence d'un modèle théorique de référence. Ce prétest a permis d'améliorer le modèle empirique, de vérifier la pertinence des dimensions à l'étude et d'ajuster le schéma d'entrevue. 
L'entrevue guidée et semi-structurée a été l'instrument privilégié pour recueillir les données de nature descriptive auprès des femmes gestionnaires sur leurs contributions. Trois thèmes figuraient sur le schéma d'analyse et respectaient une logique qui s'inscrit dans le processus classique de gestion. Le thème de culture organisationnelle, en abordant les valeurs qui sous-tendent l'organisation des services sociaux, intègre la fonction de planification. Le deuxième thème concernant le style de gestion traite des fonctions de direction et de contrôle. Le troisième, relatif au développement des services sociaux, aborde la fonction d'organisation.

L'instrument de collecte portait en première partie sur l'identification des femmes et visait à recueillir des données objectives: la situation personnelle et sociale des femmes ayant de l'influence sur leur carrière en gestion. La deuxième partie de l'instrument de collecte est un schéma d'entrevue élaboré à partir des indicateurs sous forme de questions ouvertes, regroupées selon les dimensions étudiées et respectant une progression logique.

En ce qui concerne les méthodes d'analyse des données, on s'est appuyé sur le modèle proposé par Yin (1989). La stratégie générale d'analyse basée sur le "développement d'un cadre général de description de cas» (Yin, 1989: 101)5 est apparue plus appropriée. Cette stratégie permet de cerner et de décrire les contributions des femmes gestionnaires à des services sociaux au Cameroun. Le schéma d'entrevue élaboré permet de circonscrire un cadre général de description des dix cas.

La démarche d'analyse retenue s'est faite en deux étapes. La première est la comparaison des faits au modèle de départ (patternmatching). Cette méthode est appropriée pour une étude de cas multiples. De plus, si l'étude de cas est une étude descriptive, la comparaison des faits avec le modèle initial s'avère pertinente lorsque les variables et les dimensions sont précisées avant la collecte des données, comme ce fut le cas pour cette étude.

La seconde étape d'analyse consiste à élaborer et à établir des interrelations entre les résultats obtenus d'un cas à l'autre, en retenant les constances et les différences (cross-case analysis). Il s'agit d'une analyse comparative et descriptive qui considère le milieu d'intervention (organisations publique et privée) et le niveau hiérarchique (gestionnaire fonctionnelle, gestionnaire des niveaux supérieur, intermédiaire et de premier niveau). Cette seconde étape permet de dégager les constances et les différences dans les contributions des femmes gestionnaires. 


\section{Le profil des participantes}

On trace ici le profil démographique des dix femmes gestionnaires rencontrées, leur profil scolaire et leur cheminement de carrière. L'âge des femmes gestionnaires rencontrées varie de 31 à 55 ans: trois des dix ont entre 31 et 40 ans, six ont entre 41 et 50 ans et une a plus de 51 ans.

La grande majorité des participantes à l'étude ont une formation en travail social: sept des dix femmes rencontrées ont suivi un programme d'études en service social. Trois d'entre elles possèdent un diplôme d'inspecteur des affaires sociales ou l'équivalent, deux ont une maîtrise en service social et deux autres ont un diplôme d'assistante des affaires sociales. Parmi ces dernières, l'une est sur le point $d^{\prime}$ 'obtenir un diplôme $d^{\prime}$ inspecteur des affaires sociales. Les trois autres participantes ont des formations dans des disciplines autres que le service social. Deux d'entre elles ont une formation universitaire: I'une a un Ph. D. et I'autre a une formation de deuxième cycle en administration. Enfin, la troisième femme a un diplôme d'études secondaires.

À la lumière de ce qui précède, on remarque que les femmes gestionnaires rencontrées ont un niveau de scolarisation élevé. En effet, $80 \%$ des répondantes ont un diplôme équivalent ou supérieur au baccalauréat en service social. On peut en conclure qu'au Cameroun, comme au Québec, les femmes doivent avoir un niveau d'instruction supérieur pour accéder à des postes administratifs. Ce résultat rejoint ceux d'études antérieures, notamment celle de Sutton et Moore (1985) dans laquelle on souligne que les femmes gestionnaires de nos jours sont plus instruites qu'il y a vingt ans.

Neuf femmes de notre échantillon travaillent au MINASCOF. La dixième a exercé ses fonctions dans un organisme du gouvernement avant de créer une œuvre sociale privée qu'elle dirige. Parmi les neuf femmes, sept occupent un poste de gestionnaire dans les services centraux du Ministère. La huitième dirige une institution spécialisée qui fait partie des services extérieurs du Ministère. La neuvième occupe un poste de chef de bureau dans une institution spécialisée. Cette dernière a créé et dirige une œuvre sociale privée dans laquelle elle travaille bénévolement. Elle a été interrogée en tant que gestionnaire de cette œuvre, sans que soit pris en considération son statut de fonctionnaire. Trois femmes ont exercé dans des domaines tels que l'éducation et la planification avant de se retrouver dans le service social.

Cinq femmes ont interrompu leur carrière pour poursuivre une formation à l'étranger: elles accordent donc de l'importance à la formation. Les écrits consultés signalent que l'absence de formation 
est l'un des facteurs personnels importants pouvant limiter I'accès et la mobilité professionnelle des femmes en gestion et soulignent la nécessité $d^{\prime}$ avoir une formation supérieure pour faire carrière en gestion (Bradette, 1993; Healy et al., 1990).

Les dix femmes gestionnaires rencontrées sont encore en fonction. Leurs premières expériences se traduisent par des fonctions de gestionnaire de premier niveau. Ensuite, huit d'entre elles ont gravi les échelons de la hiérarchie administrative. C'est ainsi que trois occupent actuellement le poste de gestionnaire de niveau intermédiaire au MINASCOF, trois autres sont des gestionnaires de niveau supérieur, dont deux dans les œuvres sociales privées et une dans une institution spécialisée relevant du Ministère. Deux occupent des postes de gestionnaires fonctionnelles et deux autres sont restées des gestionnaires de premier niveau depuis leur première promotion. Toutes les participantes ont plus de neuf années d'expérience de travail en gestion.

Neuf femmes exercent des activités bénévoles. Ces activités sont généralement en lien avec le service social et concernent la création ou la direction des associations de bien-être social, de personnes handicapées ou de femmes. Certaines répondantes apportent une assistance aux couples et aux personnes âgées, d'autres font partie de comités divers. Le tableau 5 ci-dessous montre les principales données socio-démographiques des femmes. On a regroupé les données par niveau hiérarchique.

\section{TABLEAU 5}

Statut civil, nombre d'enfants et niveau hiérarchique des répondantes

\begin{tabular}{cllc}
\hline $\begin{array}{l}\text { Identification } \\
\text { des répondantes }\end{array}$ & Niveau hiérarchique & Statut civil & Nombre d'enfants \\
\hline E & Fonctionnelle & Mariée & 5 \\
F & Fonctionnelle & Mariée & 4 \\
A & Supérieur & Célibataire & 0 \\
C & Supérieur & Divorcée & - \\
I & Supérieur & Mariée & 3 \\
G & Intermédiaire & Divorcée & 3 \\
H & Intermédiaire & Mariée & 3 \\
J & Intermédiaire & Mariée & 4 \\
B & Premier niveau & Divorcée & 0 \\
D & Premier niveau & Mariée & 5 \\
& & &
\end{tabular}

Les résultats du tableau 5 montrent que six des dix femmes sont mariées et ont des enfants ( 19 mois à 29 ans), trois des dix répondantes sont divorcées. Parmi ces dernières, une a des enfants et deux $n^{\prime}$ en 
ont pas. Une participante est célibataire sans enfants. La majorité de ces femmes gestionnaires ont des responsabilités familiales. De plus, parmi les sept femmes qui ont des enfants, on relève que deux ont accédé au poste de gestionnaire fonctionnelle, une au poste de gestionnaire de niveau supérieur, trois au poste de gestionnaire de niveau intermédiaire et une au poste de gestionnaire de premier niveau. Malgré le fait que dans la littérature certains auteurs (Bradette, 1993 ; York et al., 1985) soulignent que les responsabilités familiales seraient une barrière à l'évolution de la carrière des femmes en gestion, on se rend compte que, dans notre échantillon, la majorité des femmes gestionnaires sont des mères. Cela ne les a pas empêchées d'accéder à des postes de gestion. Certaines sont même montées aux hauts niveaux de la hiérarchie organisationnelle et occupent des fonctions décisionnelles, telles que gestionnaire fonctionnelle et gestionnaire de niveau supérieur. Les femmes gestionnaires rencontrées ont réussi à concilier leurs responsabilités familiales et leur carrière en gestion.

\section{La contribution des femmes gestionnaires à l'évolution de la culture organisationnelle}

Les femmes gestionnaires rencontrées ont relevé six valeurs qui, selon elles, ont guidé ou guident encore l'action sociale de MINASCOF. Ce sont par ordre d'importance: I'assistance-participation, I'assistance, la charité chrétienne, I'accessibilité, la philanthropie et le développement social. L'action sociale du MINASCOF a évolué selon ces valeurs. En général, les femmes gestionnaires rencontrées sont unanimes sur le fait que l'assistance-participation est la valeur centrale qui guide actuellement l'action sociale du MINASCOF, même si certaines femmes pensent que l'assistance reste encore une valeur à part entière dans l'organisation des services sociaux. Les avis des répondantes divergent quant à la présence de la valeur "charité chrétienne». De plus, on lui donne des sens différents. Selon l'importance qu'elles accordent à ces valeurs, il a été possible de les classer en trois groupes: les valeurs prioritaires, à savoir l'assistance-participation et I'assistance, les valeurs d'importance égale regroupant la charité chrétienne, l'accessibilité, la philanthropie et le développement social, lequel pourrait être une valeur d'avenir compte tenu de l'évolution actuelle des services sociaux camerounais.

Plus précisément, on remarque que les avis des répondantes diffèrent selon le milieu d'intervention et le niveau hiérarchique sur la présence de l'assistance et de la charité chrétienne au sein 
du système de valeurs sur lequel s'appuie l'action sociale du MINASCOF. Leurs interprétations sont parfois différentes quant au sens de ces valeurs. Par exemple, pour les participantes du secteur privé, la charité chrétienne signifie l'amour du prochain. Aussi se réfèrent-elles à cette dernière lorsqu'elles parlent de philanthropie. Pour certaines répondantes du secteur public, la charité chrétienne est une valeur individuelle qui devrait habiter chaque intervenant social. Pour d'autres, la charité est une valeur morale à promouvoir. Les valeurs en soi ne changent pas, c'est plutôt le sens qu'on leur donne qui varie.

Pour leur part, les femmes gestionnaires exerçant dans le secteur public ont identifié les six valeurs, tandis que celles œuvrant dans le secteur privé ont mentionné I'assistance-participation, I'assistance, la charité chrétienne et, dans une moindre mesure, l'accessibilité. Ces résultats s'expliqueraient par les types d'approches qui caractérisent les organisations publiques et privées. En effet, selon Godbout (1990), I'approche des organisations publiques est rationaliste, en cela qu'elles fonctionnent à partir des objectifs fixés et des moyens qu'elles prennent pour les réaliser, tandis que les organismes communautaires partent du problème et recourent à différents moyens pour le résoudre ou le diminuer. Lorsqu'on observe les valeurs privilégiées par les répondantes des deux secteurs, on note que celles des organisations privées attachent de l'importance aux valeurs qui sont en lien avec l'assistance et font intervenir la charité, tandis que celles du secteur public privilégient, dans une large mesure, I'assistanceparticipation qui constitue une valeur prioritaire déterminée par le MINASCOF.

Les participantes du secteur public intègrent la valeur prioritaire, I'assistance-participation, au moment de l'élaboration des politiques et de la planification des programmes, et elles organisent les services en ce sens. Celles du secteur privé organisent des services reflétant non seulement la valeur prioritaire établie par le MINASCOF, mais aussi la charité chrétienne et l'assistance qu'elles considèrent comme des valeurs fondamentales traduisant leur vocation d'assistance aux personnes en détresse.

On relève que l'intérêt accordé aux valeurs autres que l'assistance-participation varie en fonction de la position hiérarchique occupée. Par exemple, les répondantes de niveau intermédiaire ont mentionné les six valeurs. Ces gestionnaires occupent des postes de directrices ou de sous-directrices au MINASCOF et sont chargées de la mise en œuvre des politiques qui s'inspirent des valeurs prioritaires. Par contre, celles de niveau supérieur $n^{\prime}$ en ont mentionné 
que quatre. Les femmes gestionnaires fonctionnelles intègrent les valeurs prioritaires ou importantes dans les politiques et les programmes, celles de niveau supérieur développent des services selon ces valeurs, de même que celles de niveau intermédiaire. Les femmes gestionnaires de premier niveau intègrent les valeurs à la planification et à l'organisation des services directs à la clientèle. Leurs contributions à l'évolution de la culture organisationnelle se résument à l'élaboration des politiques et des programmes et à l'organisation et au développement des services traduisant la valeur prioritaire et les valeurs importantes.

Les répondantes du secteur public notent que la philanthropie est aussi une valeur actuelle et qu'elle pourrait prendre de plus en plus d'importance dans l'organisation des services sociaux, compte tenu du contexte de crise économique. Quant au développement social, il n'avait pas été prévu dans le modèle empirique, mais on estime qu'il mérite d'être pris en considération dans une perspective d'évolution des services sociaux.

En tenant compte de la valeur prioritaire définie par le MINASCOF, I'assistance-participation, des autres valeurs importantes (accessibilité et philanthropie) et du sens qu'elles leur donnent, les femmes gestionnaires ont décrit les gestes qu'elles font pour traduire ces valeurs dans l'organisation des services. Leur contribution à l'évolution de la culture organisationnelle dépend aussi bien des valeurs privilégiées, du milieu d'intervention que de la position occupée dans les organisations. D'une manière générale, les répondantes intègrent les valeurs ci-dessus mentionnées au moment de l'élaboration de politique, de la planification des programmes, et elles organisent des services traduisant ces valeurs. Ces contributions se rattachent à deux fonctions de gestion: la planification et I'organisation.

\section{La contribution des femmes gestionnaires au style de gestion}

La seconde variable de l'étude est le style de gestion dont les deux dimensions sont la direction et le contrôle. Pour la fonction de direction, les répondantes ont mentionné les éléments suivants: la motivation, la communication, la participation et le style de leadership.

Les répondantes ont décrit quatre formes de motivation qui émanent de leurs expériences de travail. Il s'agit de la délégation des responsabilités, de l'attribution d'indemnités, de la valorisation des apports des collaborateurs et des félicitations. Plus spécifiquement, les femmes gestionnaires des œuvres sociales privées privilégient la 
délégation des responsabilités, l'attribution d'indemnités et valorisent les apports des collaborateurs; celles des organisations publiques accordent plus d'importance à la délégation des responsabilités. Ces dernières félicitent parfois leurs collaborateurs. En somme, dans les deux milieux d'intervention, les répondantes pratiquent la délégation des responsabilités comme principale forme de motivation. Ce résultat est similaire à celui de l'analyse de Koontz et al. (1980) sur les facteurs de motivation, lequel souligne la nécessité pour le gestionnaire de rendre le travail important et significatif.

Selon le niveau hiérarchique, les femmes gestionnaires fonctionnelles font uniquement des propositions pour l'attribution des indemnités, alors que les répondantes de niveau supérieur font tous les gestes qui y sont associés. Celles de niveau intermédiaire privilégient la délégation des responsabilités. Les gestionnaires de premier niveau valorisent les apports des collaborateurs ou les félicitent verbalement.

En ce qui concerne le style de communication, les répondantes ont décrit quatre styles de communication: verbale, écrite, verticale ou horizontale. Dans I'ensemble, on remarque que dans les deux milieux d'intervention public et privé, et à tous les niveaux hiérarchiques, les femmes gestionnaires rencontrées favorisent la communication verbale. Elles la complètent par des notes écrites. Les communications orale et écrite relèvent du réseau de communication interpersonnelle que les femmes ont tendance à développer. L'étude de Bradette (1993), entre autres, révèle que la majorité des femmes gestionnaires des services sociaux estiment qu'il est important de pouvoir bien s'exprimer oralement et par écrit. En effet, l'information orale (Bergeron, 1989; Koontz et al., 1980) permet aux gestionnaires un échange rapide et complet de l'information et une réception instantanée de la rétroaction. La rétroaction pourrait être, d'ailleurs, un des éléments qui amènent les femmes à privilégier la communication interpersonnelle.

Trois formes de participation émergent des réponses des participantes: la répartition des tâches, l'incitation à la créativité des personnes et le respect de la réglementation en matière $d^{\prime}$ assiduité et de présence au travail. Les répondantes du secteur public utilisent les trois formes de participation, tandis que celles des organisations privées privilégient la répartition des tâches et l'incitation à la créativité des bénévoles.

Selon le niveau hiérarchique, les femmes gestionnaires fonctionnelles pratiquent la répartition des tâches et appliquent la réglementation. Les répondantes de niveau supérieur et celles de niveau intermédiaire favorisent la répartition des tâches et la créativité. Les 
femmes gestionnaires de premier niveau pratiquent les trois formes de participation. On observe que, aussi bien dans le secteur public que privé et à tous les niveaux hiérarchiques, les répondantes privilégient la répartition des tâches pour assurer la participation des personnes à la réalisation de la mission de l'organisation. Pour elles, il existe un lien de complémentarité entre la délégation des responsabilités et la répartition des tâches comme formes de participation.

Les répondantes se sont positionnées par rapport à quatre styles de leadership: démocratique, collégial, de laisser-faire et paternaliste. On note que leur style de leadership varie souvent en fonction du milieu d'intervention et de la position occupée. Les participantes du secteur public adoptent I'un ou I'autre des trois styles de leadership suivants : démocratique, collégial ou de laisser-faire. Celles œuvrant dans le secteur privé adoptent principalement le style démocratique. Les femmes gestionnaires rencontrées délaissent le style autocratique. Les femmes gestionnaires fonctionnelles ont un style démocratique, de même que celles de niveau supérieur; celles de niveau intermédiaire ont un style collégial et les femmes gestionnaires de premier niveau un style démocratique ou de laisser-faire.

Les styles de leadership mentionnés par les femmes sont ceux dont l'approche est fondée sur la participation, la collaboration ou les rapports égalitaires. Ces caractéristiques concernent les rapports interpersonnels, comme cela a été mentionné dans les écrits par Chernesky et Bombyk (1988: 57): ces auteurs ont conclu que les femmes gestionnaires ont un style de leadership plus humain. Toutefois, les femmes gestionnaires interrogées ne perdent pas de vue le fait qu'elles sont responsables. En effet, dans leurs commentaires, elles ont précisé que malgré le fait qu'elles délèguent l'autorité, elles coordonnent toutes les activités et veillent à ce qu'elles soient accomplies.

D'une manière générale, la contribution des femmes à l'amélioration de cette fonction est similaire et se résume à la délégation des responsabilités, à la répartition des tâches, au développement de la communication verbale et à la pratique d'un style de leadership pertinent.

Les femmes gestionnaires rencontrées ont identifié les quatre outils de contrôle suivants: I'évaluation des programmes, l'évaluation de la performance des personnes, le contrôle des ressources financières et l'évaluation des innovations en matière de services sociaux. Les répondantes du secteur public ont mentionné ces quatre éléments. Celles du secteur privé ont mentionné seulement l'évaluation des programmes et le contrôle des ressources financières. Les femmes gestionnaires fonctionnelles ont mentionné les quatre outils. Celles de niveau supérieur ont identifié l'évaluation des programmes, le 
contrôle des ressources financières et l'évaluation des innovations. Les répondantes de niveau intermédiaire ont mentionné l'évaluation des programmes, de la performance des personnes ainsi que le contrôle des ressources financières. Les femmes gestionnaires de premier niveau ont noté l'évaluation des programmes et le contrôle des ressources financières.

Trois techniques d'évaluation des programmes émergent des réponses: le processus classique d'évaluation, le développement d'un instrument d'évaluation et l'évaluation directe basée sur la qualité des objets produits ou sur l'importance de la clientèle. Les femmes gestionnaires des organisations publiques utilisent un processus classique d'évaluation, qui consiste à élaborer les plans et les programmes d'action en début d'année budgétaire et à s'en servir en fin d'année pour rédiger les rapports annuels d'exécution des activités. Cependant, certaines femmes gestionnaires du secteur public appliquent différemment ce processus classique. Pour atteindre leurs objectifs, elles font un suivi continu des programmes et les ajustent au fur et à mesure qu'elles observent des écarts. Cette approche d'évaluation va dans le sens du système de rétroaction du contrôle de gestion présenté par Koontz et al. (1980). Le processus classique d'évaluation des programmes est propre aux organisations à structure formelle comme celles du MINASCOF.

Les participantes œuvrant dans le secteur privé utilisent des mécanismes directs d'évaluation, qui consistent à se baser sur la qualité des objets produits dans le cadre de la formation artisanale ou sur l'évolution de la capacité d'accueil pour évaluer les programmes. On rencontre aussi ces mécanismes directs d'évaluation dans les organismes communautaires au Québec (Godbout, 1990). Cet auteur précise que I' "évaluation se réalise directement, par l'importance de la clientèle ou par les évaluations directes qui sont faites avec le client de toutes sortes de façons: réunions, questionnaires, etc. Dans l'évaluation, c'est le client qui a le dernier mot. On utilise peu les évaluations objectives, dites scientifiques»(Godbout, 1990: 248).

Les femmes gestionnaires fonctionnelles et celles de niveau intermédiaire privilégient les mécanismes objectifs d'évaluation des programmes. Celles de niveau supérieur utilisent des mécanismes objectifs ou directs d'évaluation des programmes. Les femmes gestionnaires de premier niveau utilisent un mécanisme objectif ou élaborent un instrument d'évaluation.

Quant à l'évaluation de la performance des personnes, rappelons que cet outil a été mentionné par les seules participantes du secteur public. Il ressort de leurs réponses qu'elles utilisent trois 
critères d'évaluation. II s'agit de la quantité des dossiers traités et de la capacité des personnes à prendre des initiatives, des bulletins de notes, du développement d'un mécanisme ou d'un instrument d'évaluation du rendement. Notons que les répondantes œuvrant dans le secteur privé $n^{\prime}$ ont pas décrit leurs expériences de travail concernant l'évaluation de la performance des personnes. Ces dernières travaillent avec des bénévoles et le critère de compétence ne revêt pas la même signification que dans le secteur public. L'étude de Godbout va dans le même sens et souligne que "Dans le modèle communautaire, la compétence repose principalement sur le fait $d^{\prime}$ avoir connu ou d'avoir résolu un problème soi-même [...] et aussi sur la valeur de la personnalité en général et sur sa capacité à être sensible aux problèmes des autres » (Godbout, 1990: 248).

Les femmes gestionnaires fonctionnelles utilisent les bulletins de notes ou développent un instrument, alors que celles de niveau intermédiaire se basent sur la quantité des dossiers traités et la capacité des personnes à prendre des initiatives.

Les gestes décrits par les femmes gestionnaires rencontrées se résument à trois mécanismes de contrôle des ressources financières: le développement d'un outil de contrôle, l'élaboration de plans d'action chiffrés et les propositions de réforme pour améliorer le contrôle des ressources financières. Les répondantes exerçant leurs fonctions dans des organisations publiques appliquent les trois mécanismes de contrôle énoncés ci-dessus, alors que celles œuvrant dans les organisations privées développent des instruments de contrôle ou élaborent des plans chiffrés adaptés à leurs besoins.

Les femmes gestionnaires fonctionnelles développent des instruments de contrôle ou font des propositions de réforme, alors que celles de niveau supérieur et celles de niveau intermédiaire élaborent des instruments ou des plans chiffrés. Les femmes gestionnaires de premier niveau mettent plutôt l'accent sur l'élaboration d'un outil de contrôle. Au total, les mécanismes de contrôle des ressources financières varient souvent selon le milieu d'intervention et le niveau hiérarchique. Dans le secteur public comme privé, et à tous les niveaux hiérarchiques, le développement d'un instrument de contrôle approprié apparaît comme le mécanisme utilisé par la plupart des répondantes.

Quant à l'évaluation des innovations en matière de services sociaux, les répondantes ont simplement décrit leur initiative sans préciser les mécanismes d'évaluation utilisés. Ces innovations se rapportent à la conception et au développement de modèles de gestion. Les écrits de certains auteurs (Braquehais,1994; Leczinska, 1982; Berlinguet, 1968) ont mis en relief l'apport des femmes gestionnaires 
des services sociaux dans l'élaboration de modèles de gestion ainsi que de plans d'organisation de services. Certaines femmes gestionnaires ont même inventé des modèles d'organisation des services sociaux (Braquehais, 1994), mais la littérature ne fait pas état des mécanismes d'évaluation de ces innovations.

Notons que I'évaluation des programmes et le contrôle des ressources financières sont les deux outils identifiés autant dans le secteur public que dans le secteur privé et à tous les niveaux hiérarchiques. La contribution des femmes gestionnaires à l'amélioration de la fonction de contrôle se résume à l'utilisation de mécanismes objectifs ou directs d'évaluation des programmes, au développement d'instruments d'évaluation du rendement, à l'élaboration de plans d'action ou au développement d'un instrument de contrôle des ressources financières.

\section{La contribution des femmes gestionnaires au développement des services sociaux}

Le développement des services sociaux est la troisième variable de notre recherche. Il porte sur le développement des organismes de distribution, des services offerts, des ressources financières et des ressources humaines du réseau des affaires sociales.

En ce qui concerne le développement des organismes de distribution, les participantes du secteur public ont mentionné les organisations publiques et privées, alors que celles des œuvres sociales privées n'ont mentionné que les organisations privées. On estime que ces dernières $\mathrm{n}^{\prime}$ ont pas de compétence en matière de création des organisations publiques. À tous les niveaux hiérarchiques, les répondantes ont identifié les organisations privées, alors que les femmes gestionnaires fonctionnelles, celles de niveau intermédiaire et celles de premier niveau ont identifié, en plus, les organisations publiques. En fonction des types d'organismes identifiés, les participantes ont décrit les gestes qu'elles font pour les développer.

Pour les organisations privées, les gestes faits par les femmes gestionnaires se résument à la création et à l'implantation de ces œuvres sociales privées et au développement de la collaboration et du partenariat avec ces organisations. Les participantes du secteur privé ont créé et implanté les œuvres sociales privées qu'elles dirigent, alors que celles du secteur public ont mis l'accent sur le développement de la collaboration et du partenariat avec le secteur privé. Ces dernières ont aussi pris part aux comités de révision de la réglementation dans ce domaine. 
Selon la position occupée, il ressort des commentaires des participantes que les gestionnaires fonctionnelles, celles de niveau intermédiaire et les gestionnaires de premier niveau ont développé la collaboration et le partenariat avec les œuvres sociales privées, alors que les répondantes de niveau supérieur ont créé et implanté les $œ u v r e s$ sociales. De tels résultats vont dans le sens de ceux de Picard (1994), de Paré (1988) et de Guerrand et al. (1978), qui ont observé que les femmes gestionnaires des services sociaux ont contribué au développement des organismes de distribution par la création, l'implantation et la direction des œuvres de bienfaisance.

En ce qui a trait aux organisations publiques, il ressort des réponses des femmes que leurs contributions au développement des organisations publiques comprennent le développement d'un modèle d'organisation pour améliorer le fonctionnement de certaines unités organisationnelles, la conception et l'implantation d'institutions spécialisées et les propositions de création de nouvelles unités organisationnelles. Ces gestes ont été faits par les seules répondantes du secteur public.

Les femmes gestionnaires fonctionnelles ont conçu un modèle d'organisation ou implanté des institutions spécialisées. Les répondantes de niveau intermédiaire et celles de premier niveau ont fait des propositions pour créer de nouvelles unités organisationnelles.

Ces résultats sont similaires à ceux généralement relevés dans les écrits sur le sujet. En effet, les femmes gestionnaires des services sociaux ont pris une part active dans les comités chargés de la transformation des œuvres sociales privées en services publics. Elles ont aussi contribué à l'implantation des services publics (Braquehais, 1994 ; Paré, 1988).

Dans l'ensemble, leurs contributions au développement des organismes de distribution se résument à la création, à l'implantation et au développement des œuvres sociales privées, à l'implantation des institutions publiques, au développement du partenariat avec le secteur privé et à l'élaboration d'un modèle d'organisation des services publics.

Pour ce qui est de la nature des services offerts les femmes gestionnaires du secteur public ont identifié les quatre catégories de services: les services généraux ou traditionnels, les services spécialisés, les services ciblés sur la clientèle et ceux répondant aux problématiques spécifiques. Celles du secteur privé ont identifié les services généraux ou traditionnels et les services spécialisés.

L'apport des femmes gestionnaires dans le développement des services généraux ou traditionnels se résume à l'organisation des services d'accueil et d'hébergement, des services d'assistance morale, 
matérielle ou financière et à l'organisation des campagnes d'information. Ce dernier aspect est développé par les répondantes exerçant dans le secteur public, alors que celles du secteur privé organisent des services d'accueil, d'hébergement et d'assistance. Les femmes gestionnaires fonctionnelles et celles de premier niveau organisent des campagnes d'information, alors que celles de niveau supérieur mettent en place tous les services mentionnés ci-dessus.

En ce qui concerne les services spécialisés, deux groupes d'action ressortent des réponses des participantes. II s'agit de l'organisation des services de rééducation et des services de formation et de l'adaptation d'une méthode d'intervention. Les femmes gestionnaires des deux milieux d'intervention organisent ces services. Celles du secteur public ont, en plus, adapté une méthode d'intervention pour une clientèle précise. À tous les niveaux hiérarchiques, les répondantes organisent les services de rééducation ou de formation. Les femmes gestionnaires de niveau intermédiaire ont en plus adapté une méthode d'éducation en milieu ouvert à une clientèle précise.

Quant aux services ciblés sur la clientèle, leurs contributions dans ce domaine se résument à l'élaboration de politiques et à I'adaptation de programmes de formation. Ces gestes sont posés par les femmes gestionnaires du secteur public occupant des postes de gestionnaires fonctionnelles.

Pour les services répondant à des problématiques spécifiques, les dires des femmes révèlent peu de contributions au développement de cette catégorie de services. Les gestes posés sont l'assistance technique pour la rédaction d'un manuel de prévention de la prostitution et le développement d'un processus d'identification des problèmes sociaux prioritaires. Les femmes gestionnaires du secteur public sont les seules à avoir développé cette catégorie des services. Elles sont des gestionnaires de premier niveau.

En somme, leurs contributions au développement des services se résument à l'élaboration de politiques appropriées, à l'organisation et au développement de divers services, à l'adaptation d'une méthode $d^{\prime}$ intervention et au développement d'un processus d'identification des problèmes sociaux prioritaires.

Quant au développement des ressources financières du réseau, les femmes gestionnaires des deux milieux d'intervention ont identifié les trois modes de financement des services sociaux suivants: les sources de financement gouvernemental, privé et international. D'une manière générale, à tous les niveaux hiérarchiques, les femmes recourent à ces trois modes de financement.

Pour ce qui est des sources de financement gouvernemental, il ressort des dires des répondantes que leur apport dans ce domaine se 
résume à trois actions: I'élaboration des budgets pertinents et réalistes, la rédaction et l'acheminement des demandes de subventions et la gestion saine des budgets. Les participantes du secteur public élaborent des budgets pertinents et pratiquent une saine gestion, alors que celles du secteur privé demandent des subventions au MINASCOF. Dans les deux cas, les répondantes disent qu'elles obtiennent les fonds sollicités. À tous les niveaux hiérarchiques, les répondantes élaborent des budgets pertinents et veillent à ce qu'ils soient utilisés à bon escient. Les femmes gestionnaires de niveau supérieur rédigent, en plus, les demandes de subventions.

En ce qui a trait aux sources de financement privé, il ressort que les gestes posés sont essentiellement la rédaction et la transmission des projets aux organisations privées nationales. Les femmes du secteur public et celles du secteur privé utilisent cette démarche. Elles disent qu'elles obtiennent souvent les fonds sollicités. De plus, certaines des femmes gestionnaires du secteur public incitent les unités organisationnelles à entreprendre des activités génératrices de revenu pour s'autofinancer. À l'exception des femmes gestionnaires de premier niveau, celles des autres niveaux hiérarchiques recherchent des fonds auprès des organisations privées nationales.

Les gestes posés pour obtenir du financement international sont principalement la rédaction, la transmission de projets aux organisations internationales et la participation aux comités chargés de négocier des financements internationaux. Les répondantes appuient ces actions par des contacts personnels. Les femmes gestionnaires qui travaillent dans le secteur public font tous ces gestes, alors que celles du secteur privé élaborent uniquement des projets. Dans les deux cas, les répondantes disent qu'elles obtiennent souvent les fonds recherchés. C'est ainsi qu'elles arrivent parfois à obtenir plusieurs financements pour le même projet. À tous les niveaux hiérarchiques, les répondantes adressent des demandes de financement aux organisations internationales. Les femmes gestionnaires fonctionnelles et celles de niveau intermédiaire ont pris part aux comités de négociation.

Au total, leurs contributions au développement des ressources financières du réseau des affaires sociales se résument à l'élaboration des budgets pertinents, à la rédaction des demandes de subventions, à l'élaboration des projets et à la pratique d'une saine gestion.

Pour ce qui est du développement des ressources humaines, les femmes du secteur public ont contribué au développement des capacités des deux catégories d'agents d'intervention: les inspecteurs des affaires sociales et les assistants et assistants adjoints des affaires sociales. Celles du secteur privé ont mentionné cette dernière 
catégorie seulement. À tous les niveaux hiérarchiques, les femmes ont contribué au développement des capacités des deux catégories de personnes mentionnées précédemment.

En ce qui concerne le développement des capacités des inspecteurs des affaires sociales, il ressort des dires des répondantes que leurs actions portent sur l'élaboration des programmes de formation, la formation, la participation aux comités d'implantation des sections de formation, la supervision des stagiaires, la direction de mémoires et la participation au jury de soutenance. Ces gestes sont posés par les femmes exerçant leurs fonctions dans le secteur public. Les femmes gestionnaires fonctionnelles ont donné de la formation, dirigé des mémoires et pris part aux comités; celles de niveau supérieur ont supervisé les stagiaires; celles de niveau intermédiaire ont donné de la formation, dirigé des mémoires et pris part au jury de soutenance de ceux-ci. Les femmes gestionnaires de premier niveau ont dirigé des mémoires et pris part aux comités. On remarque que les contributions varient en fonction de la position occupée dans l'organisation.

Quant au développement de la deuxième catégorie d'agents $d^{\prime}$ intervention, constituée par les assistants et les assistants adjoints des affaires sociales, cinq actions émergent des commentaires des répondantes: I'élaboration des programmes de formation, la formation proprement dite, la supervision des stagiaires, la formation continue et l'organisation des séminaires de formation et de recyclage. Les femmes gestionnaires du secteur public font tous ces gestes, alors que celles du secteur privé ont donné de la formation et supervisé des stagiaires. À tous les niveaux hiérarchiques, les répondantes organisent des sessions de formation continue et font la plupart des actions mentionnées ci-dessus.

En général, leurs contributions au développement des ressources humaines du réseau se résument à la participation à la mise sur pied d'une section de formation, à l'élaboration des programmes de formation, à la formation et à la supervision.

\section{CONCLUSION}

Cette étude exploratoire a permis de décrire les gestes faits par les femmes gestionnaires dans le réseau des affaires sociales au Cameroun. Les femmes rencontrées contribuent à l'évolution de la culture organisationnelle, à l'amélioration du style de gestion et au développement des services sociaux. On note une différence par rapport à leurs contributions à l'évolution de la culture organisationnelle des 
services sociaux et au développement des services offerts. Les femmes du secteur public élaborent des programmes et organisent des services en concordance avec les valeurs définies comme prioritaires par le MINASCOF. Les femmes gestionnaires des œuvres sociales privées développent des services traduisant les priorités du MINASCOF, ce qui leur permet d'obtenir des subventions, de même que les services reflétant leur vocation propre. En somme, dans les deux milieux d'intervention, les priorités du MINASCOF en matière d'organisation des services sociaux sont suivies. Ces résultats impliquent que le MINASCOF, en élaborant un cadre général d'organisation des services sociaux, doit tenir compte de la complémentarité des services en fonction des priorités qui sont différentes dans le secteur public et dans le secteur privé et en fonction des valeurs qui les sous-tendent. Au Québec, on se penche aussi actuellement sur la nature des contributions spécifiques des secteurs public et privé, sur les valeurs qui soustendent l'action de ces deux types d'organismes et sur la complémentarité de leurs actions.

Du point de vue des services développés, les résultats révèlent que les femmes gestionnaires des œuvres sociales développent des services dans des domaines qui ne sont pas couverts par le MINASCOF. Ce résultat implique que, dans le contexte actuel et compte tenu des changements à venir dans l'organisation des services sociaux au Cameroun, le MINASCOF doit revoir sa politique de partenariat qui s'est limitée, jusqu'à ce jour, à des mesures d'incitation du secteur privé pour qu'il s'implique dans l'organisation des services sociaux. Il doit définir un cadre précis de la réalisation d'un tel partenariat en associant les responsables des œuvres sociales privées à son élaboration. Ce dernier aspect a pour avantage de tenir compte de la philosophie de charité et d'assistance qui sous-tend l'organisation des services sociaux dans le secteur privé. Encore ici, des problèmes semblables se posent au Québec, alors que le gouvernement revoit sa politique de partenariat avec le secteur des services sociaux privés.

Par ailleurs, selon leur position dans I'organisation, les résultats révèlent que les femmes de notre étude ont contribué au développement de la culture organisationnelle des services sociaux, à l'amélioration du style de gestion et au développement des services sociaux. On note une concordance entre leurs contributions et les responsabilités qui sont rattachées à leurs postes. Les femmes gestionnaires de notre étude semblent être à la hauteur de leurs fonctions. C'est pourquoi, en considérant la sous-représentation des femmes, nous suggérons que le MINASCOF considère cet aspect dans ses politiques de promotion à des postes de gestion, plus précisément pour des postes de directeur ou assimilés. 
II n'a pas été possible de faire une comparaison entre les contributions des femmes gestionnaires des services sociaux au Québec et celles des femmes gestionnaires du réseau des affaires sociales au Cameroun, faute d'informations spécifiques pour déceler les similarités et les différences éventuelles entre les femmes gestionnaires des deux milieux. Cependant, on note que le modèle classique utilisé s'est facilement appliqué aux femmes gestionnaires du Cameroun, ce qui laisserait entendre que l'étude d'une telle problématique peut se faire dans plus d'un pays, plus d'un continent, en utilisant des instruments semblables, mais adaptés. De plus, les résultats de recherches québécoises sur les sujets traités dans cet article vont dans le sens des gestes faits et des apports décrits par les femmes gestionnaires du Cameroun. Enfin, le modèle de gestion classique, qui s'applique autant aux femmes qu'aux hommes, laisse croire que lorsque les femmes accèdent à des fonctions du genre, leur contribution se compare à celle des hommes qui occupent un poste équivalent. $\mathrm{Si}$ I'on a une description de la contribution des femmes, description qui a mis en évidence leur apport, on n'a cependant pas une description de la contribution spécifique des hommes et des particularités de celle-ci qui nous permettent de comparer la quantité de contributions respectives, eu égard au nombre d'hommes et de femmes gestionnaires, et la qualité, c'est-à-dire la nature différente ou semblable de ces contributions. II serait intéressant, dans une recherche ultérieure, $d^{\prime}$ investiguer sur cette question et de voir si les réponses sont semblables d'un milieu à l'autre.

\section{Notes}

1. Ministère des Affaires sociales et de la Condition féminine : Femmes et Politique (1994).

2. MINASCOF désigne le ministère des Affaires sociales et de la Condition féminine au Cameroun. Ce sigle sera utilisé dans la suite du texte. II ne sera pas nécessaire de le repréciser à chaque fois.

3. Ministère des Affaires sociales et de la Condition féminine: Planification stratégique du Minascof 1990-1992 (1988).

4. Décret $n^{\circ} 88 / 1281$ du 22 septembre 1988 portant sur l'organisation du ministère des Affaires sociales et de la Condition féminine.

5. Yin (1989 : 100-101). Dans son ouvrage, l'auteur propose deux stratégies d'analyse : la référence à des propositions théoriques et le développement d'un cadre général de description de cas. L'auteur définit cette deuxième stratégie comme une approche descriptive du cas à l'étude qui peut être utilisée lorsqu'il n'existe pas de théorie disponible. 


\section{Références bibliographiques}

BéliveAu, G. (1992). Notes de cours et recueil de textes, automne 1992, 225 pages.

BÉlIVEAU, G. et al. (1993). "Les impacts de l'informatisation des données de service social en milieu hospitalier ", Service social, vol. 42 : 103-126.

BÉLIVEAU, G. et al. (1991). Informatisation des dossiers de service social en milieu hospitalier : processus d'implantation et impacts, Laboratoire de recherche, École de service social, Université Laval, tome I, 84 pages.

BERGERON, P. (1989). La gestion moderne: théorie et cas, $2^{\mathrm{e}}$ édition. Québec : Gaëtan Morin, 565 pages.

BERLINGUET, M. (1968). "Implication de la définition des tâches sur l'efficacité des services sociaux », Service social, vol. 17, $n^{\text {os }} 1-2-3: 90-98$.

BLOUIN, H. (1994). "La réintégration des services sociaux hospitaliers : vers de nouvelles initiatives ", Intervention, $n^{\circ} 98: 26-31$.

BOISSINOT, Y. (1976). "La direction des services professionnels dans les centres de services sociaux », Service social, vol. 25, $\mathrm{n}^{\text {os }} 2-3: 29-35$.

BOISSINOT, Y. (1976). "Plan d'organisation de la direction des services professionnels au Centre de services sociaux de Québec », Service social, vol. $25, \mathrm{n}^{\text {os }} 2-3: 167-186$.

BOISSINOT, Y. (1956). "Service social et assistance publique en milieu rural », Service social, vol. 6, n 1 : 19-27.

BRADETTE, R. (1993). Les femmes cadres supérieures dans le réseau de la santé et des services sociaux : leurs discours, leurs parcours. Mémoire de maîtrise en service social, Québec, Université Laval, 163 pages.

BRAQUEHAIS, C. (1994). "La réforme du service social des directions départementales de l'action sanitaire et sociale ", Revue française de service social, $\mathrm{n}^{\text {os }} 173$ et $174: 21-29$.

CHERNESKY, R. et M. BOMBYK (1988). «Women's Ways and Effective Management ", AFFILIA, vol. 3, no $1: 48-61$.

Crener, M. et B. MONTEIL (1980). Principes de management. Sainte-Foy: Presses de I'Université du Québec, $2^{\mathrm{e}}$ édition, 532 pages.

DESLAURIERS, J.-P. (1991). Recherche qualitative. Guide pratique. Montréal : McGraw-Hill, Éd. Thema, 142 pages.

DORAIS, M. (1993). "Diversité et créativité en recherche qualitative», Service social, vol. 42, $\mathrm{n}^{\circ} 2: 7-27$.

EMGBANG EKOUDI, D. (1970). Vingt ans de service social au Cameroun et perspectives d'avenir. Mémoire de fin d'études présenté à l'École fédérale d'Éducateurs et Assistants sociaux de Betamba, 52 pages.

FAGENSON, E.A. (1990). "At the Heart of Women in Management Research : Theoretical and Methodological Approaches and Their Biases », Journal of Business Ethics, vol. 9, $\mathrm{n}^{\text {os }}$ 4-5, avril-mai $1990:$ 267-274.

GAUTHIER, B. (1984). Recherche sociale : de la problématique à la collecte des données. Sainte-Foy: Presses de I'Université du Québec, 535 pages.

Godbout, J.T. (1990). "Le communautaire et l'appareil", dans MarieMarthe T. Brault et Lise St-Jean (dir.), Entraide et associations, Québec: Institut québécois de recherche sur la culture, p. 239-259. 
Gouvernement du CAMEROUn (1993). Femmes et politique. Ministère des Affaires sociales et de la Condition féminine, p. 221-230.

GOUVERNEMENT DU CAMEROUN (1988). Décret $n^{\circ}$ 88/1281 du 22 septembre 1988 portant sur l'organisation du Ministère des Affaires sociales et de la Condition féminine, 19 pages.

GOUVERNEMENT DU QUÉBEC (1988). Rapport de la Commission d'enquête sur les services de santé et les services sociaux. Québec : Les Publications du Québec, 785 pages.

GOUVERNEMENT DU QUÉBEC (1972). Les services sociaux. Commission d'enquête sur la santé et le bien-être social, Québec : I'Éditeur officiel, tome 1, vol. VI, 380 pages.

Groulx, L.-H. (1993). Le travail social, analyse et évolution. Débats et enjeux. Laval : éd. Agence d'Arc, 297 pages.

GUBERMAN, N. (1994). "Des questions sur la culture organisationnelle des organismes communautaires", Nouvelles pratiques sociales, vol. 7, $\mathrm{n}^{\circ} 1: 45-61$.

GuERRAND, R.-H. et M.-A. Rupp (1978). Brève histoire du service social en France : 1896-1976. Toulouse : éd. Édouard Privat, 183 pages.

GUEST, D. (1980). Histoire de la sécurité sociale au Canada. P.U. ColombieBritannique et éd. Boréal (traduit de l'anglais par H. Juste, 1993).

HARDY, J.-P. (1994). "L'action sociale en 1994 », Revue française de service social, $\mathrm{n}^{\text {os }} 173$ et $174: 51-64$.

HAREL-GIASSON, F. (1990). «Femmes gestionnaires - L'actrice et I'organisation ", dans L'individu dans l'organisation : les dimensions oubliées, Jean-François Chanlat (dir.), Sillery : Les Presses de I'Université Laval, p. 406-429.

HAREL-GIASSON, F. et M. MARCHIS-MOUREN (1988). "Que nous ont appris quinze ans de fréquentation des femmes cadres? ", Actes du colloque "Tout savoir sur les femmes cadres d'ici ", Montréal: P.H.E.C., Groupes Femmes Gestion et Entreprises : 139-154.

HeAly, L., C. HAVENS et A. CHIN (1990). "Preparing Women for Human Service Administration : Building on Experience ", Administration in Social Work, vol. 14, $\mathrm{n}^{\circ} 2$ : 79-94.

KOONTZ, H. et C. O'DONNELL (1980). Management : principes et méthodes de gestion. McGraw-Hill (HD31 K82), 783 pages.

LANDRY, S. (1990). "De l'insertion des femmes dans les hautes sphères des organisations », dans Tessier et Tellier, Priorités actuelles et futures, Changement planifié et développement des organisations, tome 2 : $121-156$.

LECZINSKA, E. (1982). "Analyse systémique du modèle de gestion des services sociaux en milieu hospitalier et perspectives d'avenir », Service social, vol. 31, n' $1: 73-88$.

MALLET, L. (1994). "Éditorial, 50 ans de service social ", Revue française de service social, nos 173 et $174: 3-4$.

MAYER, R. et L.-H. Groulx (1985). Synthèse critique de la littérature sur l'évolution des services sociaux au Québec depuis 1960. Québec : Les Publications du Québec, 38 pages. 
MAYER, R. et F. OUELLET (1991). Méthodologies de recherche pour les intervenants sociaux. Québec : éd. Gaëtan Morin, 537 pages.

Miles, M.B. et A. Huberman (1984). Qualitative Data Analysis. A Sourcebook of New Method. Beverly Hills : Sage, 188 pages.

MINISTÈRE DES AFFAIRES SOCIALES ET DE LA CONDITION FÉMININE DU CAMEROUN, Programme d'activités : Exercice 1992/1993, 23 pages.

NGo NKOUTH, B. (1994). Contribution des femmes gestionnaires formées en service social à l'évolution des services sociaux au Québec de 1950 à 1990. Prétest réalisé dans le cadre du cours "Projet individuel ", 75 pages. (Non publié)

ODEWAHN, C. et F. EZELL (1992). "Attitudes Toward Women Managers in Human Service Agencies: Are They Changing?", Administration in Social Work, vol. 16, $\mathrm{n}^{\circ} 2: 45-55$.

PARÉ, S. (1988). "L'École de service social de l'Université Laval 19431988 ", dans Albert Faucher, Cinquante ans de sciences sociales à I'Université Laval : I'histoire de la faculté des sciences sociales (19391988), Québec : PUL, p. 221-249.

PASCAL, H. et C. DE ROBERTIS (1994). "Évolution de la formation des assistants sociaux », Revue française de service social, $\mathrm{n}^{\text {os }} 173$ et 174 : 107-122.

PÉRINEL, C. (1994). "Vers des partenariats renforcés? ", Revue française de service social, $\mathrm{n}^{\text {os }} 173$ et $174: 7-13$.

PERLES, J. et G.-M. SALOMON (1994). "Une page fondamentale de I'histoire du service social français ", Revue française de service social, n ${ }^{\text {os }} 173$ et $174: 65-73$.

PERRON, J. (1986). Administration sociale et services sociaux. Chicoutimi : éd. Gaëtan Morin, 285 pages.

PICARD, R. (1994). "L'assistant de service social, les services sociaux, la coordination ", Revue française de service social, $n^{\text {os }} 173$ et 174 : 15-19.

Poulin, M. (1982). "Prospective pour la gestion des services sociaux des années quatre-vingt au Québec », Service social, 1982, vol. 31, n 1 : 7-50.

SutTON, C. et K. MOORE (1985). "Executive Women - 20 Years Later», Harvard Business Review, vol. 63, $\mathrm{n}^{\circ} 5$ : 42-66.

YIN, R.K. (1989). Case Study Research : Design and Methods, Applied Social Research Methods Series, vol. 5, Sage Publications, 159 pages.

YORK, O., H. HENLEY et N. GAMBLE (1985). "Barriers to the Advancement of Women in Social Work Administration », Journal of Social Service Research, vol. 9, $\mathrm{n}^{\circ} 1: 1-15$. 